\title{
Improved Absolute Quantification using Bayesian Penalized Likelihood Reconstruction on a Digital PET/CT - Towards True Uptake Measurement
}

\section{Zhifang Wu}

First Hospital of Shanxi Medical University Department of nuclear medcine

\section{Zhixing Qin}

First Hospital of Shanxi Medical University Department of nuclear medicine

\section{Bin Huang}

First Hospital of Shanxi Medical University Department of nucleat medicine

\section{Binwei Guo}

First Hospital of Shanxi Medical University Department of nuclear medicine

\section{Xinzhong Hao}

First Hospital of Shanxi Medical University Department of Nuclear Medicine

\section{Ping Wu}

First Hospital of Shanxi Medical University Department of nuclear Medicine

\section{Bin Zhao}

First Hospital of Shanxi Medical University Department of nuclear medicine Jun Xie

Shanxi Medical University department of Biochemistry and Molecular Biology

Sijin Li ( $\sim$ lisj_nm1@sohu.com )

Department of Nuclear Medicine, First Hospital of Shanxi Medical University https://orcid.org/00000003-0361-1248

\section{Original research}

Keywords: 18F-FDG, PET-CT, Solitary pulmonary nodule

Posted Date: March 11th, 2020

DOI: https://doi.org/10.21203/rs.3.rs-16660/v1

License: (1) (1) This work is licensed under a Creative Commons Attribution 4.0 International License.

Read Full License 
Improved Absolute Quantification using Bayesian Penalized Likelihood Reconstruction on a

Digital PET/CT - Towards True Uptake Measurement

Authors: Zhifang Wu ${ }^{1,2}$, Zhixing Qin ${ }^{1}$, Bin Huang ${ }^{1}$, Binwei Guo ${ }^{1}$, Xinzhong Hao ${ }^{1}$, Ping Wu ${ }^{1}$,

Bin $\mathrm{Zhao}^{1}$, Jun $\mathrm{Xie}^{3}$, Sijin $\mathrm{Li}^{1,2}$

1 Department of Nuclear Medicine, First Hospital of Shanxi Medical University, Taiyuan, Shanxi,

P.R. China

2 Molecular Imaging Precision Medical Collaborative Innovation Center, Shanxi Medical University, Taiyuan, Shanxi, P.R. China

3 Department of Biochemistry and Molecular Biology, Shanxi Medical University, Taiyuan, Shanxi, P.R. China

\section{Corresponding author(s): Jun Xie, Sijin Li}

Prof. Jun Xie $(\bowtie)$, Department of Biochemistry and Molecular Biology, Shanxi Medical University, Taiyuan, Shanxi, P.R. China. E-mail: xiejun102030@163.com

Prof. Sijin Li $(\bowtie)$, Department of Nuclear Medicine, First Hospital of Shanxi Medical University, No. 85 South Jiefang Road, Taiyuan, 030001, Shanxi, P.R. China. E-mail: lisjnm123@163.com. Tel: 86-351-4639430, Fax: 86-351-4048123 


\section{Abstract}

\section{Background}

To evaluate the quantification accuracy, we measured the recovery coefficient (RC) and contrast recovery (CR) in a phantom study and used it as a guide in clinical evaluation about digital positron emission tomography -computed tomography (PET/CT).

\section{Methods}

The RC and CR of the PET images reconstructed with 4 different methods [ordered subsets expectation maximization (OSEM), time of flight (TOF), TOF-point spread function (PSF), bayesian penalized likelihood (BPL)] were measured in the phantom study. And, SUVmax and SUVmean (the maximum and mean of the standardized uptake values, SUVs) of 75 small pulmonary nodules (sub-centimeter group: $<10 \mathrm{~mm}$ and medium size group: $10-25 \mathrm{~mm}$ ) from 26 patients were measured with those methods. For lesions smaller than 3 times of the spatial resolution, partial-volume-effect correction (PVC) based on RC values was performed.

\section{Results}

BPL enables higher RC and CR values, which can improve quantification accuracy compared with other methods $(\mathrm{P}<0.05)$, except for the RC of $37 \mathrm{~mm}$ sphere $(\mathrm{P}>0.05)$. The SUVs of sub-centimeter fludeoxyglucose (FDG)-avid pulmonary nodules with BPL illustrated highly significant difference from those reconstructed with other algorithms $(\mathrm{P}<0.001)$. However, reconstruction algorithms had less impact on SUVs measured in medium group ( $>>0.05)$. With the PVC, highly significant difference $(\mathrm{P}<0.001)$ still existed in the SUVmean measured on BPL compared with those measured on the other 3 algorithms, although PVC had the least effect on BPL.

\section{Conclusions}

BPL on the digital PET/CT demonstrated improved quantification accuracy. For small pulmonary nodules, PVC is important.

\section{Keywords}

18F-FDG, PET-CT, Solitary pulmonary nodule

\section{Backgroud}

Quantitative PET imaging shows growing importance in the early-stage disease diagnosis $[1,2]$ and treatment response evaluation [3,4] by noninvasively monitoring the physiological and 
pathological processes in vivo. In the development of PET imaging system, many research efforts have been devoted to reinforce the power of quantification accuracy and small lesion detectability [5, 6]. On one hand, due to the partial volume effect caused by the limited spatial resolution of conventional PET systems, the radiotracer uptake is usually underestimated when measuring a lesion smaller than the 3 times of the spatial resolution [7]. On the other hand, the traditional iterative image reconstruction algorithm, e.g. ordered-subsets expectation maximization (OSEM), is not able to reach full convergence because the noise in the image grows with each iteration, and hence there exists a compromise between iteration and noise resulting in partial convergence [8,9]. Therefore, it is particularly difficult to assess the true metabolic activity of the small lesions, such as sub-centimeter pulmonary nodules or lymph nodes. Many studies demonstrated the challenges of evaluating sub-centimeter nodules, for instance, the differential diagnostic sensitivity became lower for determining malignancy of such nodules than that of larger ones [10-12], and even false-negative findings can be generated [13]. The visibility of small lesions and quantification accuracy facilitates the improved staging, treatment planning, response monitoring and prognostic estimation, and they are of importance for clinical diagnostic confidence and patient management [14].

Generally, it is impossible to know the true uptake of a lesion in vivo. In order to access the quantification accuracy, the most reliable method is to measure the recovery coefficient (RC) in the phantom study, which gives the true activity by calculating the ratio of measured activity to the true one determined by the dose calibrator [15]. Contrast recovery (CR) is another important index reflecting the true uptake ratio in the lesion and background by showing the ratio between measured sphere-to-background activity and known sphere-to-background activity [16]. The BPL based reconstruction algorithm (known as Q.Clear, GE Healthcare) on a digital PET/CT system (Discovery MI, GE Healthcare) has shown significant advantages over conventional reconstruction algorithm (OSEM) in photomultiplier tube (PMT)-based PET/CT scanners [17-20]. It has been revealed that the new BPL-based reconstructed PET images provided significant increases in signal-to-background, signal-to-noise (SNR) ratios and SUVs, with greatly enhanced visual sensitivity for assessing small pulmonary nodules [21, 22], liver metastasis [23] and mediastinal nodes in non-small cell lung cancer [24]. Although SUVs measured from the BPL reconstruction algorithm achieved higher values, whether this SUV elevation reflects higher quantification accuracy need to be verified.

Therefore, we evaluated the quantification accuracy of BPL-based reconstruction on SiPM-based 
PET/CT system with a phantom study and performed a clinical study on pulmonary nodules based on the results of the phantom study.

\section{Methods}

\section{Phantom}

A National Electrical Manufacturers Association (NEMA) image quality phantom [25] was utilized in this study with 6 spheres filled with $13.2 \mathrm{kBq} / \mathrm{mL}{ }^{18} \mathrm{~F}-\mathrm{NaF}$ in a 4-to-1 ratio (sphere to background activity concentration). Recovery coefficient (RC) and contrast recovery (CR) was measured using the following equations (Eq.1-2).

$$
\begin{aligned}
& R C=\frac{A_{M}}{A_{K}} \times 100 \% \\
& C R=\frac{\frac{A_{M}}{A_{B}}-1}{C-1} \times 100 \%
\end{aligned}
$$

Where $A_{M}$ is the measured activity (in $\mathrm{kBq} / \mathrm{mL}$ ) in each sphere delineated on CT images; $\mathrm{A}_{B}$ is the measured activity in the background. $A_{K}$ is the known activity (in $\mathrm{kBq} / \mathrm{mL}$ ) in the sphere; $C$ is the known ratio of activity in the sphere to the background (that is $4: 1$ in the study).

\section{PET/CT imaging}

${ }^{18}$ F-FDG PET/CT scans were performed on a SiPM-based digital PET/CT system (Discovery MI, GE Healthcare, USA) equipped with BPL (Q.Clear, GE Healthcare) reconstruction algorithm with an axial field of view $25 \mathrm{~mm}$. Before scanning, all the patients fasted for at least $6 \mathrm{~h}$ with blood glucose level being lower than $200 \mathrm{mg} / \mathrm{dL}$. Then, the patients received $2.96-3.70 \mathrm{MBq} / \mathrm{kg}$ of ${ }^{18} \mathrm{~F}-\mathrm{FDG}$ and rested for approximately 60 min after injection. The CT images were acquired using a slice thickness of $3.75 \mathrm{~mm}$, pitch of $0.984,120 \mathrm{kVp}, 60-150 \mathrm{~mA}$ modulation with a noise index of 18 , rotation time of 0.5 s. PET imaging was conducted with $3 \mathrm{~min} / \mathrm{bed}$ in List-mode that allows for postpone PET image reconstructions. The phantom was scanned three times with the same PET/CT imaging parameters.

\section{Image reconstruction}

All the phantom and clinical PET data were reconstructed using 4 different algorithms, namely OSEM (2 iterations, 17 subsets, 6.4-mm post-filter cutoff), TOF: OSEM+TOF (time-of-flight, with same OSEM parameters except for 3 iterations), TOF-PSF: OSEM+TOF+PSF (point-spread-function, same OSEM parameters except for 3 iterations), BPL: OSEM+TOF+PSF+BPL $(\beta=350)$, where $\beta$ is defined in the BPL objective function as below, 


$$
\hat{x}=\arg \max _{x \geq 0} \sum y_{i} \log \left[P_{x}\right]_{i}-\left[P_{x}\right]_{i}-\beta R(x)
$$

where $x$ is the image estimate; $i$ is the pixel index; $y_{i}$ represents the measured PET coincidence data; $P$ is the system geometry matrix; $\beta$ is the penalization factor; $\mathrm{R}(\mathrm{x})$ is the penalty to control noise.

\section{Clinical evaluation}

For the retrospective nature of this study, informed consent is waived. We retrospectively reviewed the imaging data of all patients with lung nodules who underwent ${ }^{18} \mathrm{~F}$-FDG PET/CT scans between March and July of 2018 in our hospital. A total number of 75 Pulmonary nodules from 26 patients (20 males and 6 females; median age 66 years old, range 36-82 years old; median height 170 $\mathrm{cm}$, range $155-183 \mathrm{~cm}$; median weight $61.5 \mathrm{~kg}$, range $40-100 \mathrm{~kg}$ ) with long-axis diameter of $\leq 25 \mathrm{~mm}$ on CT lung window were enrolled. The nodules sized under $10 \mathrm{~mm}$ were categorized as sub-centimeter group, while the medium size group was from 10 to $25 \mathrm{~mm}$. The reconstructed PET images were processed using the PET Volume Computer-Assisted Reading (PET VCAR, GE Healthcare) on an Advantage Workstation 4.7 (GE Healthcare), which allows automatic segmentation based on iterative adaptive algorithm [26]. We measured SUVmean and SUVmax of small pulmonary nodules.

\section{PVC for pulmonary nodules}

A linear regression was generated based on the relationship of RC and sphere diameter (measured on CT images) in each reconstruction method. The partial volume effect correction was performed on all pulmonary nodules, based on the small sized nature of them, by dividing the corresponding RC. The PVC percentage increase, $\% \Delta$ SUVmeans, were calculated as the difference between PVE corrected SUVmeans and the original ones (Eq. 4).

$$
\% \Delta \text { SUVmeans }=\frac{P V C \text { SUVmeans }- \text { SUVmeans }}{\text { SUVmeans }} \times 100 \%
$$

\section{Statistical analysis}

Statistical analysis was conducted using SPSS Statistics 25.0 (IBM Co., New York, USA). Kruskal Wallis $\mathrm{H}$ test was utilized to analyze differences in phantom data (RC and $\mathrm{CR})$ and in clinical data (SUVs) among the 4 reconstruction algorithms. Mann-Whitney U test was used to compare the SUVmean difference before and after PVC. P value $<0.05$ was consider as statistically significant difference, while $\mathrm{P}<0.001$ was taken as highly significant difference.

\section{Results}

\section{Phantom}



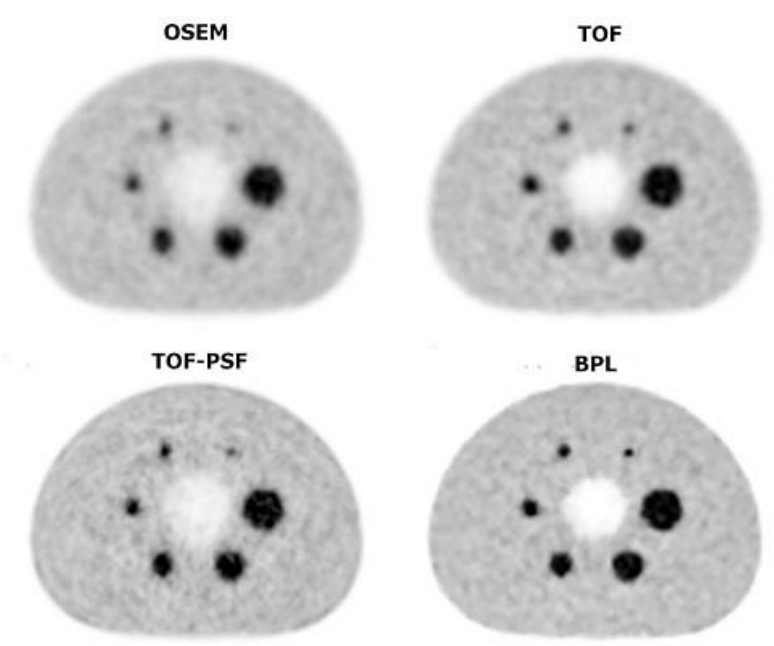

Figure 1 Comparison of PET reconstruction methods for NEMA phantom with 6 spheres (diameter 10-37 mm) filled with $13.2 \mathrm{kBq} / \mathrm{mL} 18 \mathrm{~F}-\mathrm{NaF}$ in a 4-to-1 contrast ratio. The mean uptake values $(\mathrm{Bq} / \mathrm{mL})$ of the 6 spheres (in order from $10 \mathrm{~mm}$ to $37 \mathrm{~mm}$ ) for 4 reconstructions were as follow: 4.12, 5.66, 6.57, 7.52, 9.03, 9.78 for OSEM, 5.36, 7.25, 8.32, 8.77, 9.62, 10.24 for TOF, 5.76, 7.86, 8.84, 8.96, 9.67, 10.47 for TOF-PSF, $6.70,9.09,9.54,9.62,10.99,11.15$ for BPL. All the PET images are displayed on SUV scale $0-8$.

From the visual assessment (Figure 1), as more advanced reconstruction techniques (from OSEM

121 to BPL-based reconstruction) introduced into the PET reconstruction, the overall image quality improved. Thanks to the BPL-based reconstruction algorithm, the smallest sphere (diameter=10 $\mathrm{mm}$ )

123 was particularly outstanding with clearer boundaries, meanwhile, reduced background noise was also achieved compared with other 3 reconstruction methods.

Table 1 The average uptake Am, recovery coefficient and contrast recovery determined from 6 hot spheres

\begin{tabular}{|c|c|c|c|c|c|c|}
\hline \multirow{2}{*}{ Sphere } & \multicolumn{3}{|c|}{ OSEM } & \multicolumn{3}{|c|}{ TOF } \\
\hline & $\mathrm{A}_{\mathrm{m}}(\mathrm{Bq} / \mathrm{ml})$ & $\mathrm{RC}(\%)$ & $\mathrm{CR}(\%)$ & $\mathrm{A}_{\mathrm{m}}(\mathrm{Bq} / \mathrm{ml})$ & $\mathrm{RC}(\%)$ & $\mathrm{CR}(\%)$ \\
\hline $10 \mathrm{~mm}$ & $4.34 \pm 0.25$ & $40.0 \pm 1.9$ & $35.5 \pm 1.88$ & $5.31 \pm 0.25$ & $50.3 \pm 1.90$ & $43.4 \pm 1.17$ \\
\hline $13 \mathrm{~mm}$ & $6.00 \pm 0.34$ & $60.0 \pm 2.5$ & $49.2 \pm 2.26$ & $7.15 \pm 0.10$ & $64.3 \pm 0.70$ & $58.4 \pm 1.20$ \\
\hline $17 \mathrm{~mm}$ & $7.04 \pm 0.42$ & $60.0 \pm 3.2$ & $57.7 \pm 2.00$ & $8.16 \pm 0.17$ & $72.0 \pm 1.30$ & $66.7 \pm 1.33$ \\
\hline $22 \mathrm{~mm}$ & $7.89 \pm 0.32$ & $70.0 \pm 2.4$ & $64.7 \pm 1.32$ & $8.62 \pm 0.34$ & $75.5 \pm 2.60$ & $70.4 \pm 1.60$ \\
\hline $28 \mathrm{~mm}$ & $9.29 \pm 0.26$ & $80.0 \pm 2.0$ & $76.1 \pm 0.19$ & $9.70 \pm 0.24$ & $83.7 \pm 1.80$ & $79.3 \pm 0.49$ \\
\hline \multirow[t]{3}{*}{$37 \mathrm{~mm}$} & $10.00 \pm 0.24$ & $90.0 \pm 1.8$ & $82.0 \pm 0.40$ & $10.22 \pm 0.25$ & $87.7 \pm 1.90$ & $83.6 \pm 0.34$ \\
\hline & & TOF-PSF & & & BPL & \\
\hline & $\mathrm{A}_{\mathrm{m}}(\mathrm{Bq} / \mathrm{ml})$ & $\mathrm{RC}(\%)$ & $\mathrm{CR}(\%)$ & $\mathrm{A}_{\mathrm{m}}(\mathrm{Bq} / \mathrm{ml})$ & $\mathrm{RC}(\%)$ & $\mathrm{CR}(\%)$ \\
\hline
\end{tabular}

\begin{tabular}{lllllll}
\hline $10 \mathrm{~mm}$ & $5.66 \pm 0.17$ & $50.0 \pm 1.30$ & $46.1 \pm 0.70$ & $6.80 \pm 0.45$ & $59.0 \pm 1.40$ & $53.2 \pm 1.89$ \\
$13 \mathrm{~mm}$ & $7.73 \pm 0.16$ & $70.0 \pm 1.20$ & $63.0 \pm 1.04$ & $9.04 \pm 0.13$ & $79.0 \pm 1.00$ & $74.4 \pm 0.59$
\end{tabular}




\begin{tabular}{lllllll}
$17 \mathrm{~mm}$ & $8.69 \pm 0.19$ & $70.0 \pm 1.50$ & $70.6 \pm 1.32$ & $9.57 \pm 0.42$ & $82.0 \pm 1.20$ & $78.7 \pm 1.77$ \\
$22 \mathrm{~mm}$ & $8.80 \pm 0.34$ & $73.9 \pm 2.60$ & $71.7 \pm 1.43$ & $9.49 \pm 0.21$ & $83.0 \pm 1.60$ & $78.0 \pm 0.54$ \\
$28 \mathrm{~mm}$ & $9.76 \pm 0.23$ & $82.8 \pm 1.80$ & $79.5 \pm 0.66$ & $10.76 \pm 0.21$ & $91.7 \pm 1.60$ & $88.5 \pm 0.78$ \\
$37 \mathrm{~mm}$ & $10.45 \pm 0.26$ & $87.4 \pm 1.90$ & $85.2 \pm 0.15$ & $10.92 \pm 0.24$ & $92.9 \pm 1.80$ & $89.8 \pm 0.52$ \\
\hline
\end{tabular}

A
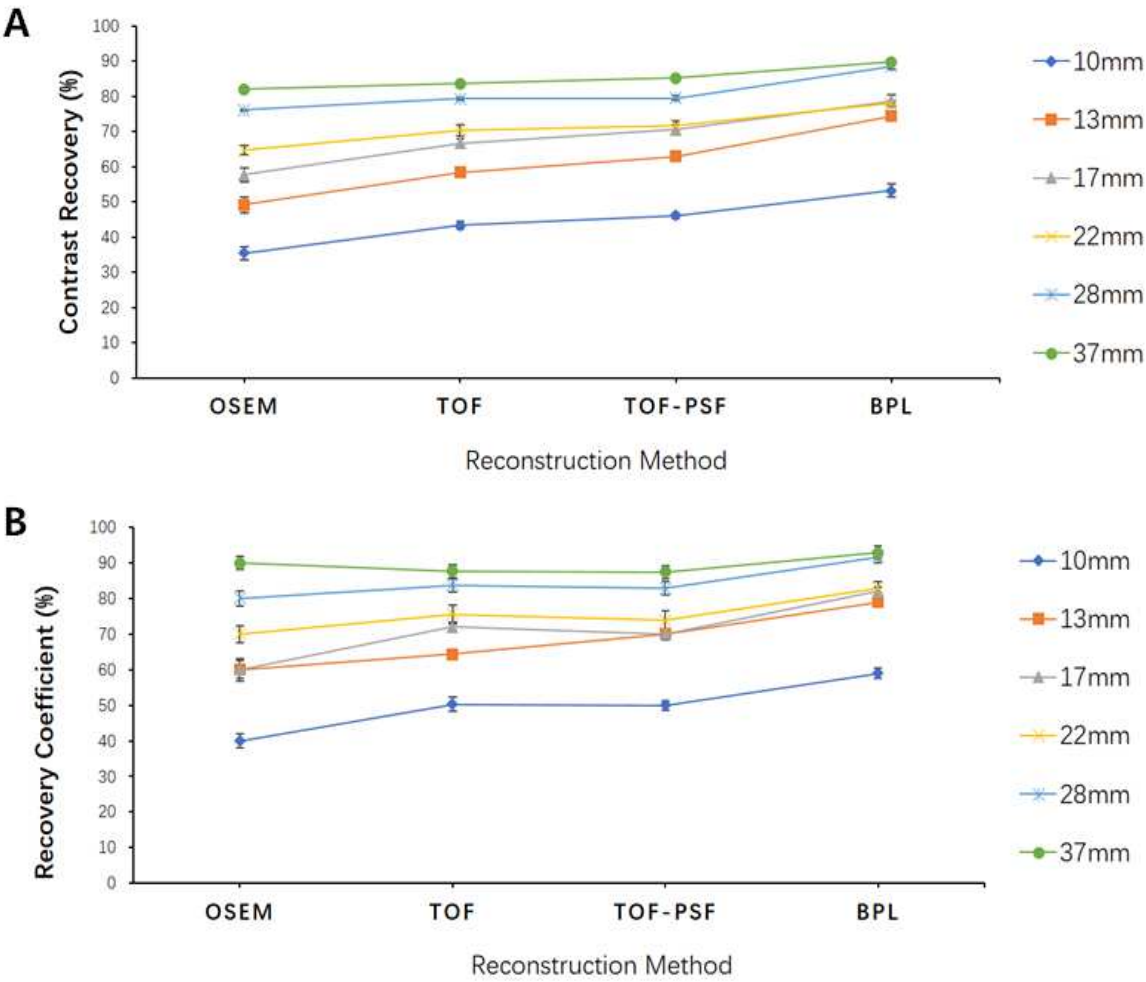

for 6 hot spheres (diameter 10-37 mm) filled with $13.2 \mathrm{kBq} / \mathrm{mL}$ Fluoride ions in a 4-to-1 contrast ratio.

Table 1 and Figure 2 has shown that $\mathrm{A}_{\mathrm{m}}(\mathrm{Bq} / \mathrm{ml}), \mathrm{CR}(\%)$ and $\mathrm{RC}(\%)$ increased gradually in the order from OSEM, TOF, TOF-PSF to BPL. The average $\mathrm{A}_{\mathrm{m}}$ and RC of 5 spheres $(10 \mathrm{~mm}-28 \mathrm{~mm})$ using BPL method were significantly higher than those of other reconstruction methods $(\mathrm{P}<0.05)$ while the biggest sphere $(37 \mathrm{~mm})$ showed no significant difference among reconstruction methods $(\mathrm{P}>$ 0.05). The CR values of all hot spheres using BPL method had higher values than that of the other reconstruction algorithms $(\mathrm{P}<0.05)$ (Table 2). The RC values of 6 hot spheres calculated from BPL method reached up to 59.0\% (10 mm), 79.0\% (13 mm), 82.0\% (17 mm), 83.0\% (22 mm), 91.7\% (28 $\mathrm{mm})$ and $92.9 \%(37 \mathrm{~mm})$ while CR values reached up to $53.2 \%(10 \mathrm{~mm}), 74.4 \%(13 \mathrm{~mm}), 78.7 \%$ (17 
A

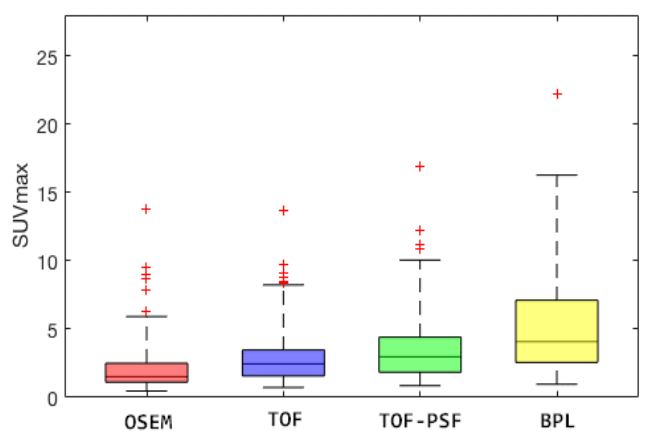

B

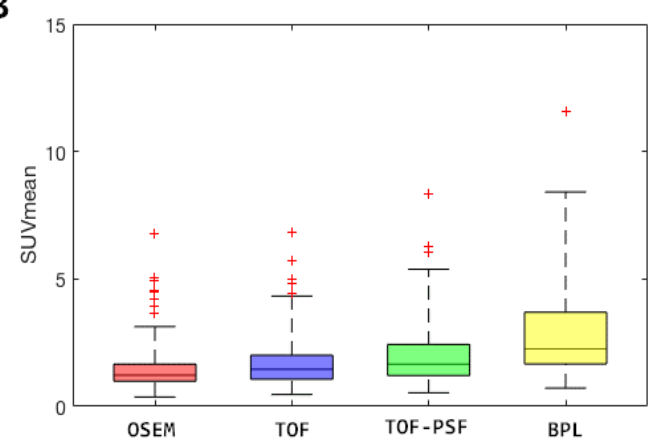

Figure 3 The box plots of (A) SUVmax and (B) SUVmean measurements of 75 pulmonary nodules on PET images reconstructed with different methods. Highly statistically significant differences (noted as $* * * *$, $\mathrm{P}$ values $<0.001$ ) in the SUVmax and SUVmean were shown between PET images reconstructed with BPL and each of the other 3 methods (OSEM, TOF, and TOF-PSF).

Table 2 The significance difference in 6 spheres among 4 reconstruction methods ( $\mathrm{P}$ value)

\begin{tabular}{cccccccc}
\hline \multirow{2}{*}{ Parameters } & \multicolumn{3}{c}{ Sphere } \\
\cline { 2 - 7 } & $10 \mathrm{~mm}$ & $13 \mathrm{~mm}$ & $17 \mathrm{~mm}$ & $22 \mathrm{~mm}$ & $28 \mathrm{~mm}$ & 0,031 \\
\hline $\mathrm{A}_{\mathrm{m}}$ & 0,018 & 0,016 & 0,016 & 0,022 & 0,064 & 0,023 & 0,016 \\
$\mathrm{CR}$ & 0,016 & 0,016 & 0,016 & 0,022 & 0,031 & 0,075 \\
$\mathrm{RC}$ & 0,018 & 0,016 & 0,016 & 0,022 & & $0 . \mathrm{mm}$ \\
\hline
\end{tabular}

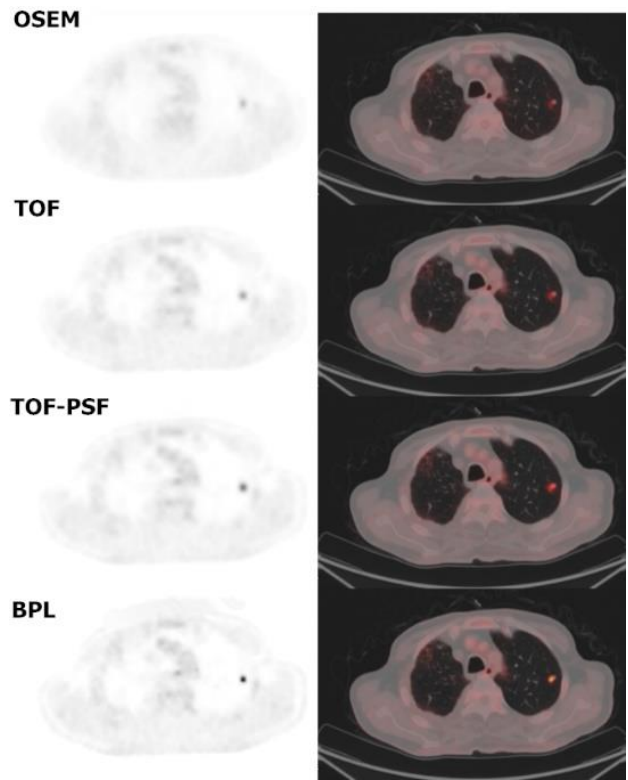

Figure 4 Illustration of PET images of a pulmonary nodule (long-axis diameter $7.2 \mathrm{~mm}$ ) reconstructed with different algorithms. The SUVmax of OSEM, TOF, TOF-PSF, BPL reconstructed images were 1.93, 2.62, 3.9, 7.28 respectively. The SUVmean were $0.99,1.42,1.87,3.46$ respectively. All the PET images are displayed on SUV scale $0-8$. 
An illustration of the same pulmonary nodule (long-axis diameter $7.2 \mathrm{~mm}$ ) on the PET images 146 reconstructed using different reconstruction algorithms was shown in Figure 4 and the lesion became 147 clearer when BPL reconstruction was used. The SUVmax and SUVmean calculated from all the small 148 pulmonary nodules $(n=75)$ increased gradually in the order from OSEM, TOF, TOF-PSF to BPL and 149 SUVs determined from BPL method were significantly higher than those from other reconstruction 150 methods $(\mathrm{P}<0.001$ for SUVmax and SUVmean) (Figure 3).

\section{1}

Table 3 The impact of different reconstruction methods on SUVs of small pulmonary nodules before/after PVC

\begin{tabular}{|c|c|c|c|c|c|c|c|c|c|c|c|}
\hline \multirow{2}{*}{ Groups } & \multirow{2}{*}{ Size $(\mathrm{mm})$} & \multicolumn{5}{|c|}{ SUVmax } & \multicolumn{5}{|c|}{ SUVmean } \\
\hline & & OSEM & TOF & TOF-PSF & BPL & $P$ value & OSEM & TOF & TOF-PSF & BPL & $P$ value \\
\hline \multicolumn{12}{|l|}{$\leq 10 \mathrm{~mm}$} \\
\hline $\mathrm{n}=57$ & $5.03 \pm 1.79$ & $1.58 \pm 0.82$ & $2.26 \pm 1.07$ & $2.76 \pm 1.39$ & $4.32 \pm 2.46$ & 0,000 & $1.18 \pm 0.53$ & $1.45 \pm 0.69$ & $1.63 \pm 0.76$ & $2.50 \pm 1.32$ & 0,000 \\
\hline \multicolumn{12}{|l|}{$10-25 \mathrm{~mm}$} \\
\hline \multirow[t]{2}{*}{$\mathrm{n}=18$} & $15.12 \pm 4.32$ & $5.52 \pm 3.56$ & $6.13 \pm 3.44$ & $7.23 \pm 4.24$ & $8.77 \pm 5.65$ & 0,128 & $3.04 \pm 1.70$ & $3.31 \pm 1.68$ & $3.76 \pm 2.00$ & $4.92 \pm 2.90$ & 0,050 \\
\hline & & \multicolumn{5}{|c|}{ Corr SUVmax } & \multicolumn{5}{|c|}{ Corr SUVmean } \\
\hline & & OSEM & TOF & TOF-PSF & BPL & $P$ value & OSEM & TOF & TOF-PSF & BPL & $P$ value \\
\hline \multicolumn{12}{|l|}{$\leq 10 \mathrm{~mm}$} \\
\hline $\mathrm{n}=57$ & $5.03 \pm 1.79$ & $3.71 \pm 1.79$ & $4.37 \pm 1.93$ & $5.08 \pm 2.41$ & $6.68 \pm 3.63$ & 0,000 & $2.78 \pm 1.15$ & $2.79 \pm 1.24$ & $2.99 \pm 1.31$ & $3.86 \pm 1.98$ & 0,006 \\
\hline \multicolumn{12}{|l|}{$10-25 \mathrm{~mm}$} \\
\hline $\mathrm{n}=18$ & $15.12 \pm 4.32$ & $9.55 \pm 6.40$ & $9.48 \pm 5.48$ & $10.99 \pm 6.69$ & $11.68 \pm 7.80$ & 0,771 & $5.32 \pm 3.15$ & $5.14 \pm 2.73$ & $5.72 \pm 3.18$ & $6.56 \pm 4.02$ & 0,711 \\
\hline
\end{tabular}

154 SUVmax and SUVmean determined from sub-centimeter pulmonary nodules had highly significant 155 difference among different reconstruction techniques $(\mathrm{P}<0.001)$ while the medium size group showed 156 no significant difference $(\mathrm{P}>0.05)$. 

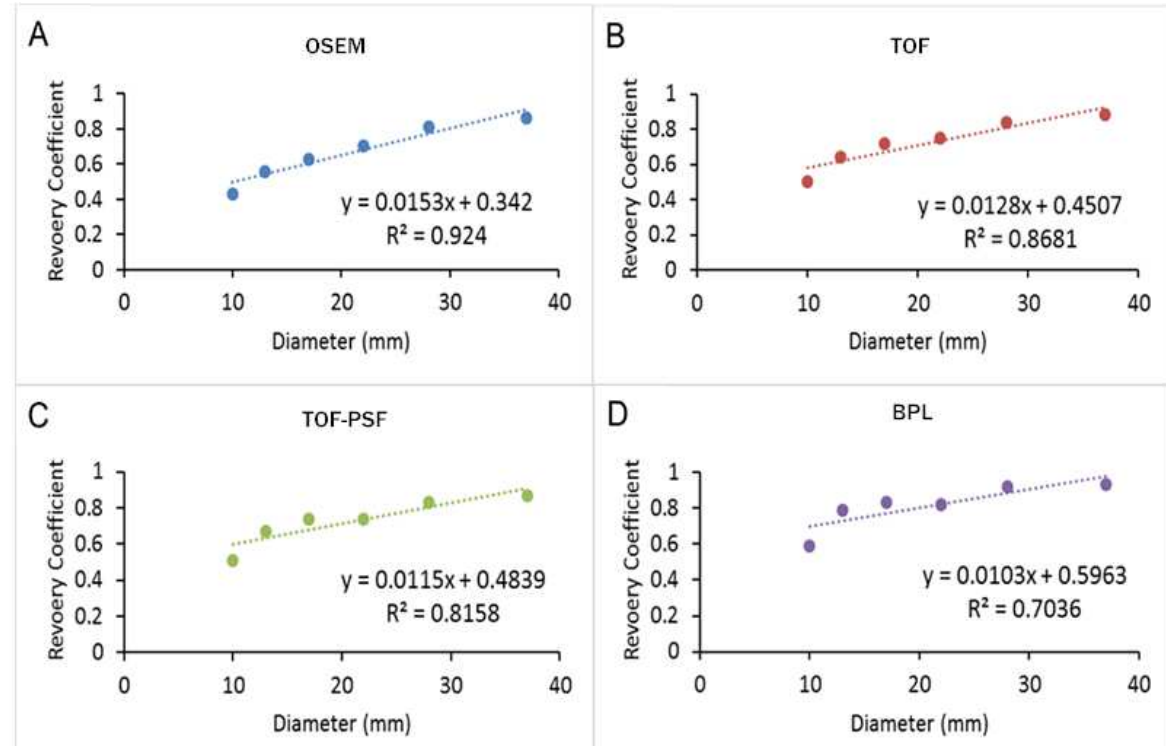

Figure 5 Recovery coefficients calculated based on PET images reconstructed with different algorithms (A) OSEM, (B) TOF, (C) TOF-PSF, (D) BPL, showing as a function of measured sphere diameter on CT images. The equations and R2 values of linear fittings between recovery coefficient and measured sphere diameter were presented.

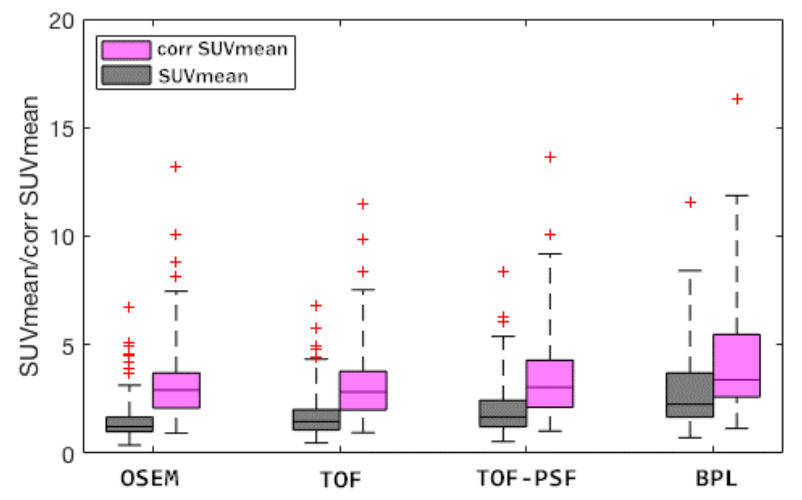

Figure 6 The box plots of SUVmean and PVE corrected SUVmean (corr SUVmean) of 75 pulmonary nodules on PET images reconstructed with different methods. After the PVE correction, the SUVmean increases represented by $\% \triangle \mathrm{SUV}$ mean were $124.5 \%, 85.3 \%, 77.1 \%$, and $49.4 \%$ for OSEM, TOF, TOF-PSF and BPL, respectively. 

SUVmean and the corr SUVmean in each reconstruction algorithm $(\mathrm{P}<0.001$ for all), but also between BPL method and the other three ones in corr SUVmean $(\mathrm{P}<0.001$ for all). However, differences in SUVs measured from 4 reconstruction methods were not significant for the medium size group (Table 3, $\mathrm{P}=0.771$ for $\mathrm{SUVmax}$ and $\mathrm{P}=0.711$ for SUVmean).

\section{Discussion}

A reliable and precise measurement of radiopharmaceutical uptake is more and more important in PET, particularly for differential diagnosis, treatment planning and therapy response evaluation. It is still challenging to achieve accurate quantification of radiotracer uptake in the small lesions due to the partial convergence in current reconstruction algorithms and the partial volume effect, which usually underestimate SUV. In this study, we demonstrated the values of using BPL reconstruction algorithm on the SiPM-based PET/CT platform for improving quantification accuracy and image quality. The BPL-based reconstruction revealed considerable enhancement towards true uptake compared with the other reconstruction methods, especially in sub-centimeter pulmonary nodules.

The BPL-reconstructed images generated the highest RC and CR compared to the other 3 methods (Figure 2), suggesting BPL-based reconstruction improved the quantification accuracy of PET imaging approaching the true uptake. However, it was found that the RC difference among 4 reconstruction methods was not significant $(\mathrm{P}>0.05)$ in the largest sphere $(37 \mathrm{~mm})$. The reason for this is, under the same iteration number, the degree of convergence of OSEM based reconstruction algorithms were higher in large subjects, which can be seen in the Figure 2 that larger spheres always had higher RC values compared with smaller spheres regardless of reconstruction methods used.

For the quantification of pulmonary nodules, significant increases in the SUVmax and SUVmean were observed when reconstructed with BPL-based algorithm compared to the other 3 methods (Figure 3), and the increases for those of sub-centimeter nodules were significantly greater than those of the medium size group (Table 3, $\mathrm{P}<0.001$ ) By comparing the changes of SUVs among different reconstruction methods in the medium size group, increments can be found but was not statistically significant (Table 3, P > 0.05). This was because, firstly, medium size group was closer to full convergence on routine OSEM based algorithms compared to the sub-centimeter group; secondly, the number of nodules enrolled in medium size group was inadequate $(n=18)$ to reach statistical 
significance. Similarly, Teoh et al. also showed that the greatest improvement was found in malignancy detection in nodules $\leq 10 \mathrm{~mm}$ compared with larger ones [21]. This concluded the BPL-reconstruction algorithm exerted more profound impact on the SUV measurement of small pulmonary nodules, which potentially facilitated the enhancement of the lesion visibility and detectability, especially for sub-centimeter lesions.

Another impact factor on SUV quantification accuracy of small lesions is the partial volume effect, which also underestimate the true uptake. In our phantom study, RC was calculated to reveal the direct relationship between the measured and true radioactivity. Then the association between RC values and sphere diameter measured on $\mathrm{CT}$ images was established for each reconstruction method. The linear regression was performed and good regression coefficient was achieved (Figure 5). Based on the equations and the measured nodule size in CT images, we corrected the partial volume effect to improve the SUV measurement accuracy of all pulmonary nodules. This RC-based PVE correction approach is on the assumptions that the lesion has a regular spherical shape and uniform distribution of radioactivity [27]. Due to the limited size range of phantom sphere being from $10 \mathrm{~mm}$ to $37 \mathrm{~mm}$, the PVE correction for those nodules either smaller than $10 \mathrm{~mm}$ or larger than $37 \mathrm{~mm}$ should be careful. In addition, in Figure 6, the number of outlier values decreased in the order from OSEM, TOF, TOF+PSF to BPL which indicated the improvement on the SUV reliability. A few previous studies have demonstrated this method for PVE correction [11, 28, 29], for instance, Srinivas et al. created a 'lookup table' for determining approximate RC values for lesions in different sizes and disease regions [27]. Our finding on the relationship between RC and sphere diameter showed consistency with their result. Future elaborated phantom experiment designs should be dedicated to investigating in different clinical imaging scenarios, with different lesion size, lesion shape, image contrast and radioactivity level. It should be noted that this PVE correction method is equipment-specific, which means different PET scanners may have different RC-diameter behaviors.

In this study, image reconstruction parameters were chosen based on the routine clinical protocols in our hospital. As small lesions are more challenge clinically, hard to reach full convergence on the routine OSEM based algorithms and are heavily affected by PVE, nodules with size under $25 \mathrm{~mm}$ were enrolled.

The respiratory motion during PET imaging may compromise the spatial resolution, resulting in smearing or blurring effect of PET images, which would eventually affect SUV measurement. Existing 
researches suggested that respiratory movement leads to overestimation of radiotracer-avid target

227 volume and reduction in the SUV due to the recorded number of coincidence events tends to distribute in a larger volume [30]. The respiratory gating system can be employed in the future study to facilitate the improvement on spatial resolution and quantification accuracy, especially when radiotherapy treatment planning is desired [31-33].

Furthermore, the small pulmonary nodules in our study were not histopathological verified. The relationship between malignancy and SUV measurement of pulmonary nodules under BPL reconstruction need to be investigated, which could benefit differential diagnosis based on quantitative PET/CT imaging.

\section{Conclusion}

In our phantom and clinical studies, the BPL-based reconstruction algorithm combined with SiPM-based digital PET/CT platform significantly improved the quantification accuracy towards the true uptake by accessing RC and $\mathrm{CR}$ values, which potentially promotes the diagnostic confidence and treatment response evaluation with PET/CT imaging especially for the sub-centimeter pulmonary nodules. For small lesions, PVE corrections is essential.

(1)

$$
\text { nodules. For small lesions, PVE corrections is essential. }
$$


$257 \quad$ List of abbreviation

258 BPL Bayesian penalized likelihood

259 CR Contrast recovery

260 CT Computed tomography

261 FDG Fluorodeoxyglucose

262 NEMA National Electrical Manufacturers Association

263 OSEM Ordered-subsets expectation maximization

264 PET Positron emission tomography

265 PMT Photomultiplier tube

266 PSF Point spread function

267 PVC Partial-volume-effect correction

268 PVE Partial-volume-effect

269 RC Recovery coefficient

270 SBR Signal-to-background

271 SiPM Silicon-photomultiplier

272 SNR Signal-to-noise

273 SUV Standardized uptake value

274 SUVmax Maximum standardized uptake value

275 SUVmean Mean standardized uptake value

276 TOF Time-of-flight

277 


\section{Declaration}

\section{Ethics approval and consent to participate}

For the retrospective nature of this study, informed consent is waived.

\section{Consent for publication}

Not applicable.

\section{Availability of data and material}

The datasets used and analysed during the current study are available from the corresponding author on reasonable request.

\section{Funding}

This work was supported by the National Natural Science Foundation (No. 81571716, 81671724), the Science and Technology Planning Project of Shanxi Province (No. 2015091017, 201703D421020).

\section{Authors' contributions}

Zhifang Wu carried out the research methods, participated in design of this study and drafted the manuscript. Zhixing Qin, Bin Huang, Bin Zhao and Binwei Guo reconstructed the PET images with 4 different methods and calculated the RC and CR. Ping Wu and Xinzhong Hao participated in the design of the study and performed the statistical analysis. Xie Jun and Sijin Li conceived of the study, and participated in its design and coordination and helped to draft the manuscript. All authors read and approved the final manuscript.

\section{Acknowledgements}

The authors would like to thank advanced application specialists of GE Healthcare China for their assists during the study.

\section{Competing Interests}

The authors declare that they have no competing interests 


\section{Reference}

1. Pastorino U, Bellomi M, Landoni C, De Fiori E, Arnaldi P, Picchio M, Pelosi G, Boyle P, Fazio F (2003) Early lung-cancer detection with spiral CT and positron emission tomography in heavy smokers: 2-Year results. Lancet 362:593-597

2. Sironi S, Buda A, Picchio M, Perego P, Moreni R, Pellegrino A, Colombo M, Mangioni C, Messa C, Fazio F (2006) Lymph Node Metastasis in Patients with Clinical Early-Stage Cervical Cancer: Detection with Integrated FDG PET/CT. Radiology 238:272-279

3. Ben-Haim S, Ell P (2009) 18F-FDG PET and PET/CT in the Evaluation of Cancer Treatment Response. J Nucl Med. https://doi.org/10.2967/jnumed.108.054205

4. Schwarz JK, Grigsby PW, Dehdashti F, Delbeke D (2009) The Role of 18F-FDG PET in Assessing Therapy Response in Cancer of the Cervix and Ovaries. J Nucl Med. https://doi.org/10.2967/jnumed.108.057257

5. Tong S, Alessio AM, Kinahan PE (2010) Image reconstruction for PET/CT scanners: past achievements and future challenges. Imaging Med 2:529-545

6. van der Vos CS, Koopman D, Rijnsdorp S, Arends AJ, Boellaard R, van Dalen JA, Lubberink M, Willemsen ATM, Visser EP (2017) Quantification, improvement, and harmonization of small lesion detection with state-of-the-art PET. Eur J Nucl Med Mol Imaging 44:4-16

7. Soret M, Bacharach SL, Buvat I (2007) Partial-Volume Effect in PET Tumor Imaging. J Nucl Med 48:932-945

8. Jaskowiak CJ, Bianco J a, Perlman SB, Fine JP (2005) Influence of reconstruction iterations on 18F-FDG PET/CT standardized uptake values. J Nucl Med 46:424-8

9. Parvizi N, Franklin JM, McGowan DR, Teoh EJ, Bradley KM, Gleeson F V. (2015) Does a novel penalized likelihood reconstruction of 18F-FDG PET-CT improve signal-to-background in colorectal liver metastases? Eur J Radiol 84:1873-1878

10. Nomori H, Watanabe K, Ohtsuka T, Naruke T, Suemasu K, Uno K (2004) Evaluation of F-18 fluorodeoxyglucose (FDG) PET scanning for pulmonary nodules less than $3 \mathrm{~cm}$ in diameter, with special reference to the CT images. Lung Cancer 45:19-27

11. Alkhawaldeh K, Bural G, Kumar R, Alavi A (2008) Impact of dual-time-point 18F-FDG PET imaging and partial volume correction in the assessment of solitary pulmonary nodules. Eur $\mathbf{J}$ Nucl Med Mol Imaging 35:246-252 
12. Khalaf M, Abdel-Nabi H, Baker J, Shao Y, Lamonica D, Gona J (2008) Relation between nodule size and 18F-FDG-PET SUV for malignant and benign pulmonary nodules. J Hematol Oncol 1:1-8

13. Iwano S, Ito S, Tsuchiya K, Kato K, Naganawa S (2013) What causes false-negative PET findings for solid-type lung cancer? Lung Cancer 79:132-136

14. Herder GJ, Golding RP, Hoekstra OS, Comans EF, Teule GJ, Postmus PE, Smit EF (2004) The performance of $18 \mathrm{~F}$-fluorodeoxyglucose positron emission tomography in small solitary pulmonary nodules. Eur J Nucl Med Mol Imaging 31:1231-1236

15. Jentzen W, Weise R, Kupferschläger J, Freudenberg L, Brandau W, Bares R, Burchert W, Bockisch A (2008) Iodine-124 PET dosimetry in differentiated thyroid cancer: Recovery coefficient in 2D and 3D modes for PET(/CT) systems. Eur J Nucl Med Mol Imaging 35:611623

16. Hsu DFC, Ilan E, Peterson WT, Uribe J, Lubberink M, Levin CS (2017) Studies of a Next-Generation Silicon-Photomultiplier-Based Time-of-Flight PET/CT System. J Nucl Med $58: 1511-1518$

17. Lindström E, Sundin A, Trampal C, Lindsjö L, Ilan E, Danfors T, Antoni G, Sörensen J, Lubberink M (2018) Evaluation of penalized likelihood estimation reconstruction on a digital time-of-flight PET/CT scanner for ${ }^{18}$ F-FDG whole-body examinations. J Nucl Med jnumed.117.200790

18. Wagatsuma K, Miwa K, Sakata M, Oda K, Ono H, Kameyama M, Toyohara J, Ishii K (2017) Comparison between new-generation SiPM-based and conventional PMT-based TOF-PET/CT. Phys Medica 42:203-210

19. I. S, L. B, S. P, N. H, S. S, G. D, S.S. G, A. I (2018) Initial experience with a SiPM-based PET/CT scanner: influence of acquisition time on image quality. EJNMMI Phys. https://doi.org/10.1186/s40658-018-0207-x

20. Baratto L, Park SY, Hatami N, Davidzon G, Srinivas S, Gambhir SS, Iagaru A (2017) 18F-FDG silicon photomultiplier PET/CT: A pilot study comparing semi-quantitative measurements with standard PET/CT. PLoS One 12:1-13

21. Teoh EJ, McGowan DR, Bradley KM, Belcher E, Black E, Gleeson F V. (2016) Novel penalised likelihood reconstruction of PET in the assessment of histologically verified small 
pulmonary nodules. Eur Radiol 26:576-584

22. Howard BA, Morgan R, Thorpe MP, Turkington TG, Oldan J, James OG, Borges-Neto S (2017) Comparison of Bayesian penalized likelihood reconstruction versus OS-EM for characterization of small pulmonary nodules in oncologic PET/CT. Ann Nucl Med 31:623-628

23. Teoh EJ, McGowan DR, Macpherson RE, Bradley KM, Gleeson F V. (2015) Phantom and Clinical Evaluation of the Bayesian Penalized Likelihood Reconstruction Algorithm Q.Clear on an LYSO PET/CT System. J Nucl Med 56:1447-1452

24. Teoh EJ, McGowan DR, Bradley KM, Belcher E, Black E, Moore A, Sykes A, Gleeson F V. (2016) 18F-FDG PET/CT assessment of histopathologically confirmed mediastinal lymph nodes in non-small cell lung cancer using a penalised likelihood reconstruction. Eur Radiol 26:4098-4106

25. Association NEM, NU N (2013) 2-2012 performance measurement of positron emission tomography. Rosslyn, VA NEMA

26. Fox JJ, Autran-Blanc E, Morris MJ, et al (2011) Practical approach for comparative analysis of multilesion molecular imaging using a semiautomated program for PET/CT. J Nucl Med $52: 1727-1732$

27. Srinivas SM, Dhurairaj T, Basu S, Bural G, Surti S, Alavi A (2009) A recovery coefficient method for partial volume correction of PET images. Ann Nucl Med 23:341-348

28. Suzawa N, Ito M, Qiao S, Uchida K, Takao M, Yamada T, Takeda K, Murashima S (2011) Assessment of factors influencing FDG uptake in non-small cell lung cancer on PET/CT by investigating histological differences in expression of glucose transporters 1 and 3 and tumour size. Lung Cancer 72:191-198

29. Geworski L, Knoop BO, de Cabrejas ML, Knapp WH, Munz DL (2000) Recovery correction for quantitation in emission tomography: a feasibility study. Eur J Nucl Med Mol Imaging 27:161-169

30. Erdi YE, Nehmeh SA, Pan T, et al (2004) The CT motion quantitation of lung lesions and its impact on PET-measured SUVs. J Nucl Med 45:1287-92

31. Nehmeh SA, Erdi YE, Ling CC, et al (2002) Effect of respiratory gating on reducing lung motion artifacts in PET imaging of lung cancer. Med Phys 29:366-371

32. Nehmeh SA, Erdi YE, Ling CC, Rosenzweig KE, Schoder H, Larson SM, Macapinlac HA, 
Squire OD, Humm JL (2002) Effect of Respiratory Gating on Quantifying PET Images of Lung Cancer. J Nucl Med 43:876-881

33. Werner MK, Parker JA, Kolodny GM, English JR, Palmer MR (2009) Respiratory gating enhances imaging of pulmonary nodules and measurement of tracer uptake in FDG PET/CT. Am J Roentgenol 193:1640-1645 


\section{Figures}
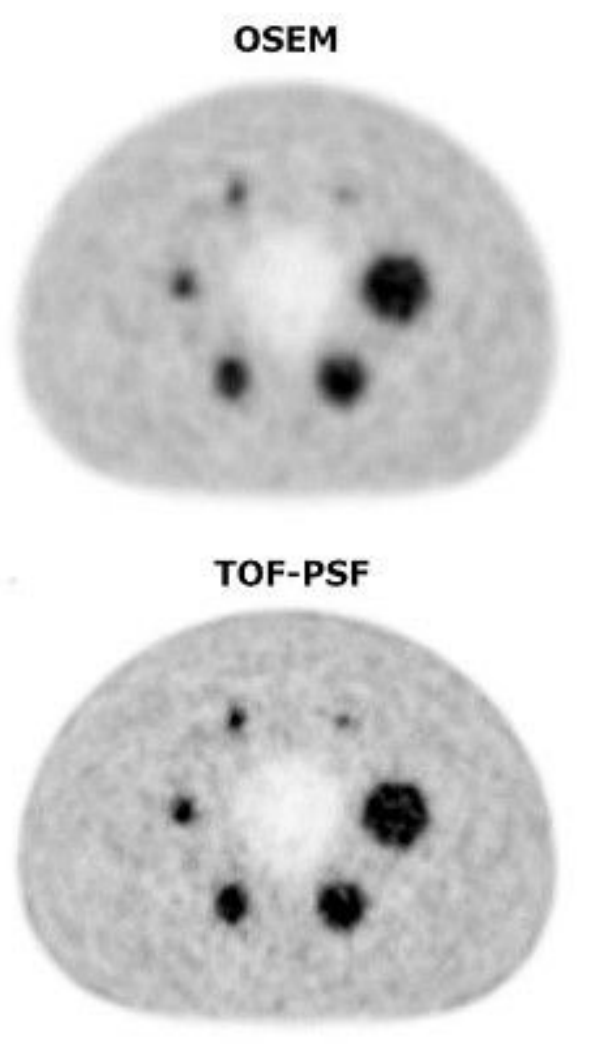
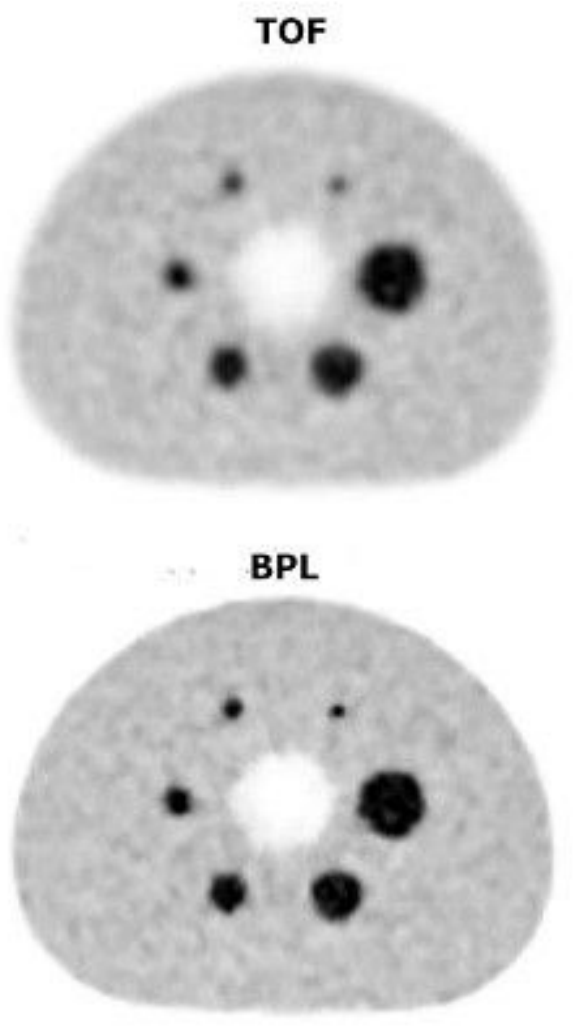

Figure 1

Comparison of PET reconstruction methods for NEMA phantom with 6 spheres (diameter 10-37 mm) filled with $13.2 \mathrm{kBq} / \mathrm{mL} 18 \mathrm{~F}-\mathrm{NaF}$ in a 4-to- 1 contrast ratio. The mean uptake values $(\mathrm{Bq} / \mathrm{mL})$ of the 6 spheres (in order from $10 \mathrm{~mm}$ to $37 \mathrm{~mm}$ ) for 4 reconstructions were as follow: 4.12, 5.66, 6.57, 7.52, 9.03, 9.78 for OSEM, 5.36, 7.25, 8.32, 8.77, 9.62, 10.24 for TOF, 5.76, 7.86, 8.84, 8.96, 9.67, 10.47 for TOF-PSF, $6.70,9.09,9.54,9.62,10.99,11.15$ for BPL. All the PET images are displayed on SUV scale 0-8. 
A

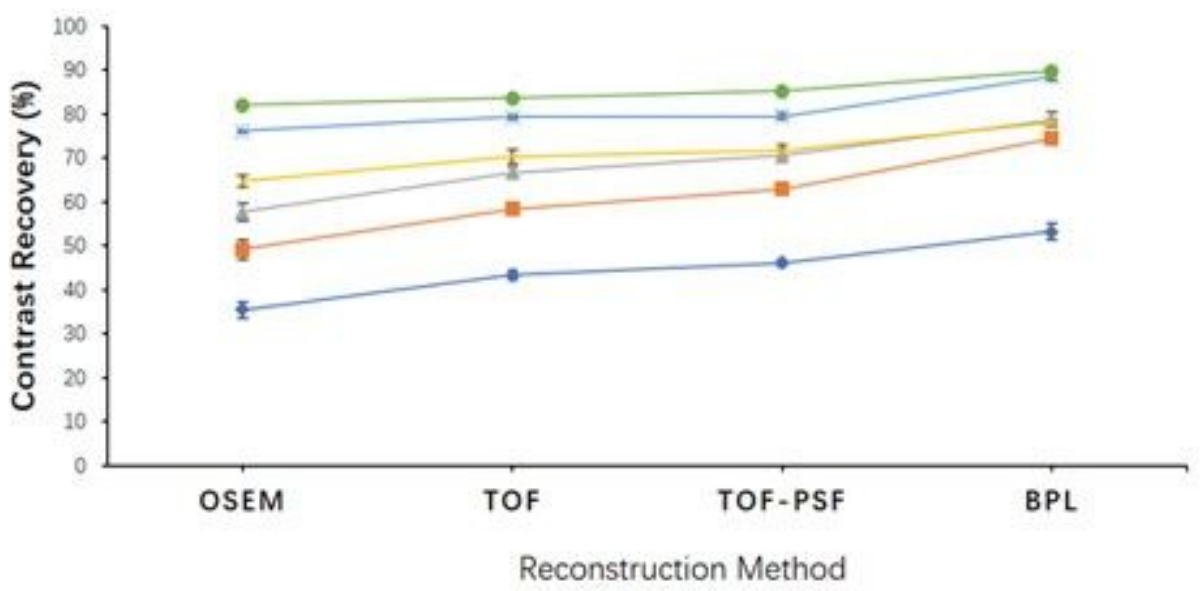

$--10 \mathrm{~mm}$

$-13 \mathrm{~mm}$

$₫-17 \mathrm{~mm}$

$-22 \mathrm{~mm}$

$-28 \mathrm{~mm}$

$-37 \mathrm{~mm}$

B

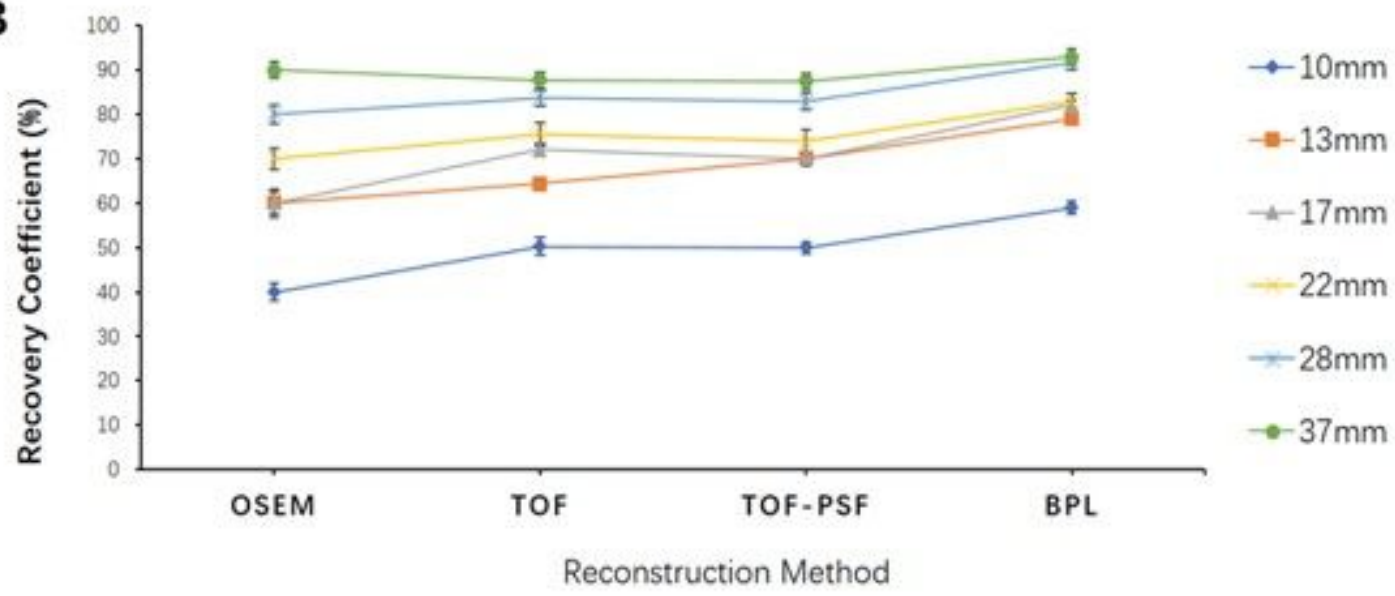

\section{Figure 2}

Comparison of recovery coefficient (A) and contrast recovery (B) among PET reconstruction methods for 6 hot spheres (diameter 10-37 mm) filled with $13.2 \mathrm{kBq} / \mathrm{mL}$ Fluoride ions in a 4-to-1 contrast ratio.

A

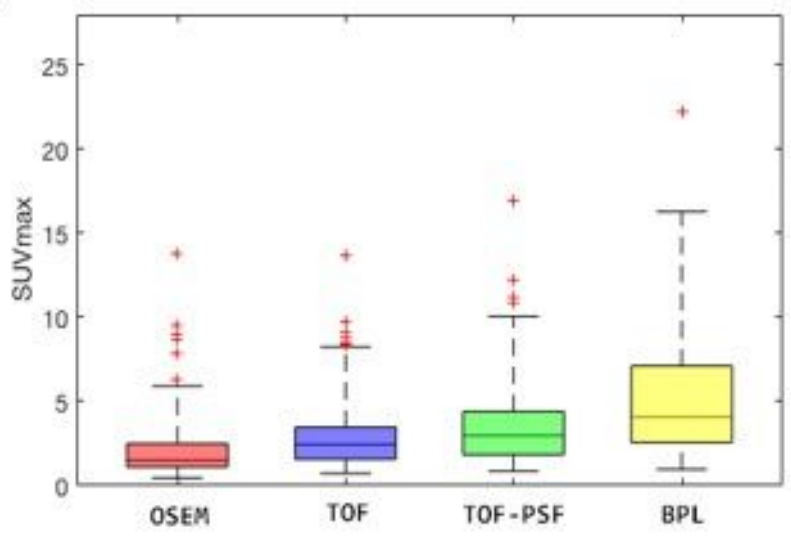

B

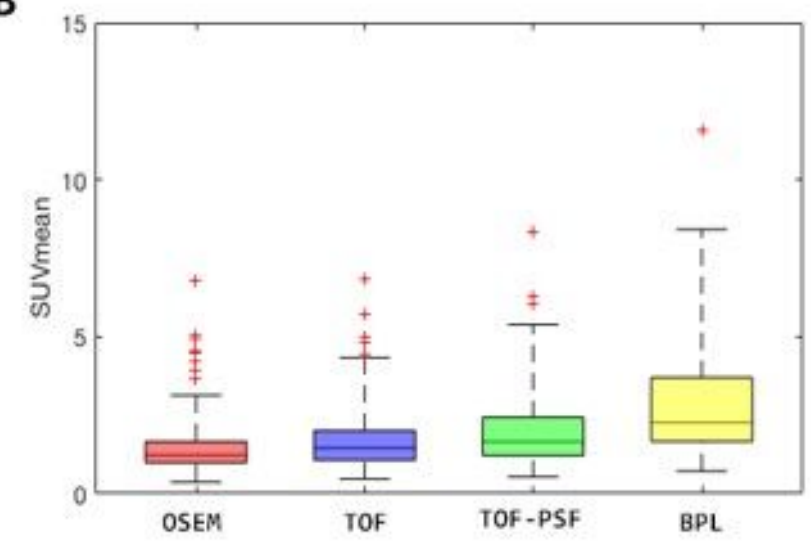

Figure 3 
The box plots of (A) SUVmax and (B) SUVmean measurements of 75 pulmonary nodules on PET images

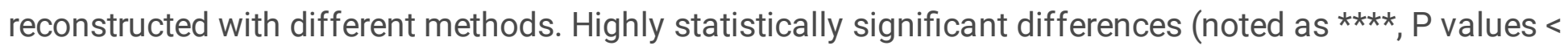
0.001) in the SUVmax and SUVmean were shown between PET images reconstructed with BPL and each of the other 3 methods (OSEM, TOF, and TOF-PSF).

\section{OSEM}

\section{TOF}

\section{TOF-PSF}

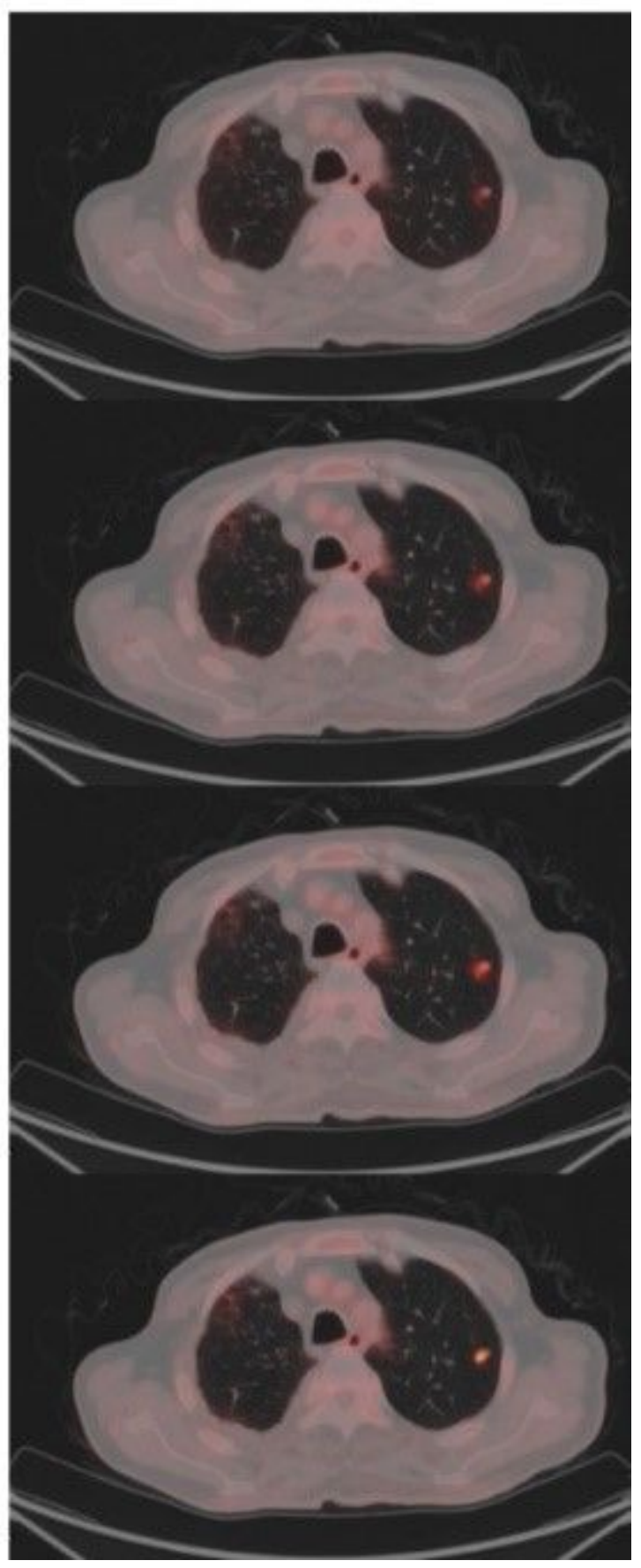

\section{BPL}

\section{Figure 4}

Illustration of PET images of a pulmonary nodule (long-axis diameter $7.2 \mathrm{~mm}$ ) reconstructed with different algorithms. The SUVmax of OSEM, TOF, TOF-PSF, BPL reconstructed images were 1.93, 2.62, $3.9,7.28$ respectively. The SUVmean were $0.99,1.42,1.87,3.46$ respectively. All the PET images are displayed on SUV scale 0-8. 


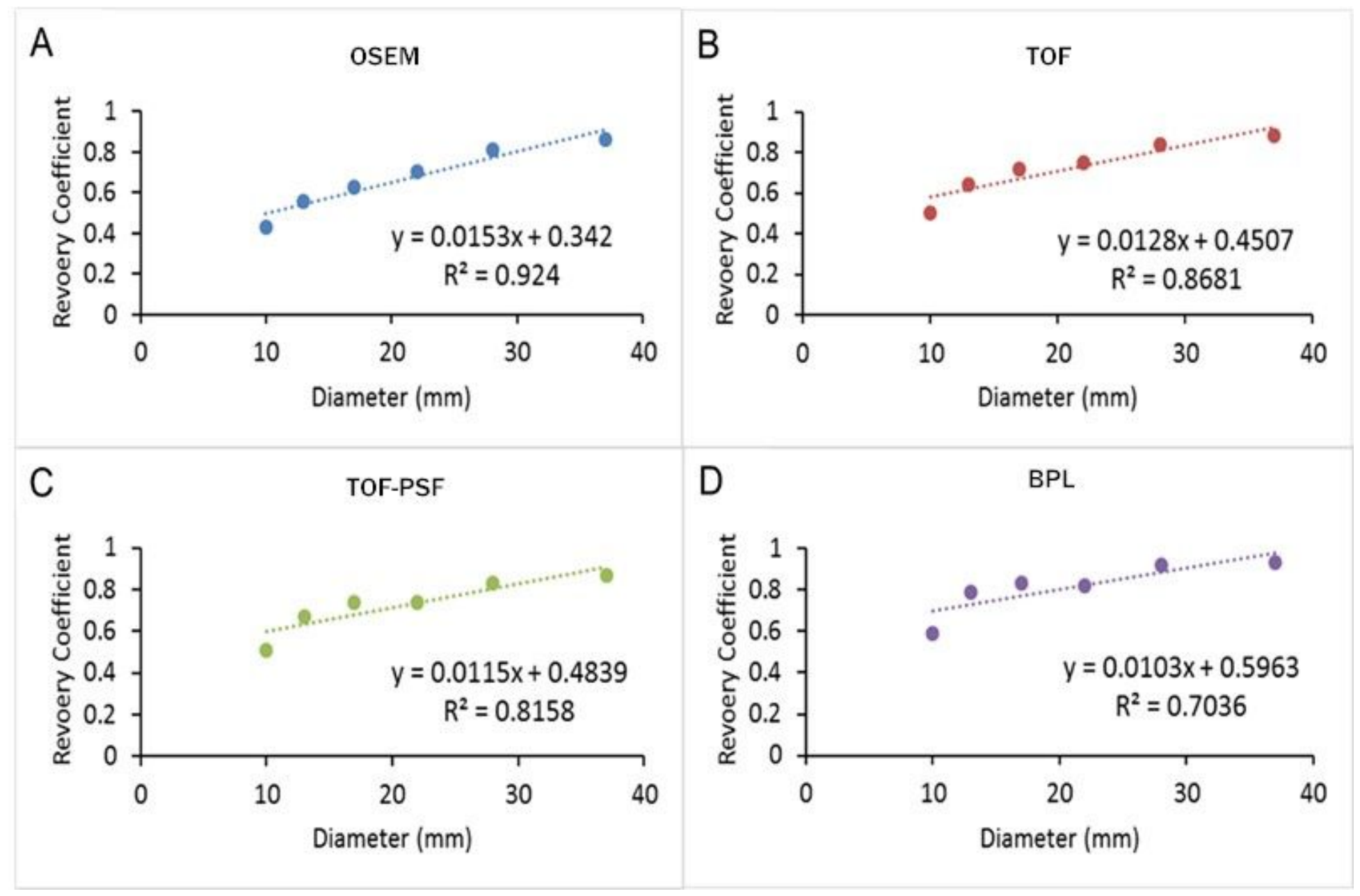

Figure 5

Recovery coefficients calculated based on PET images reconstructed with different algorithms (A) OSEM, (B) TOF, (C) TOF-PSF, (D) BPL, showing as a function of measured sphere diameter on CT images. The equations and R2 values of linear fittings between recovery coefficient and measured sphere diameter were presented. 


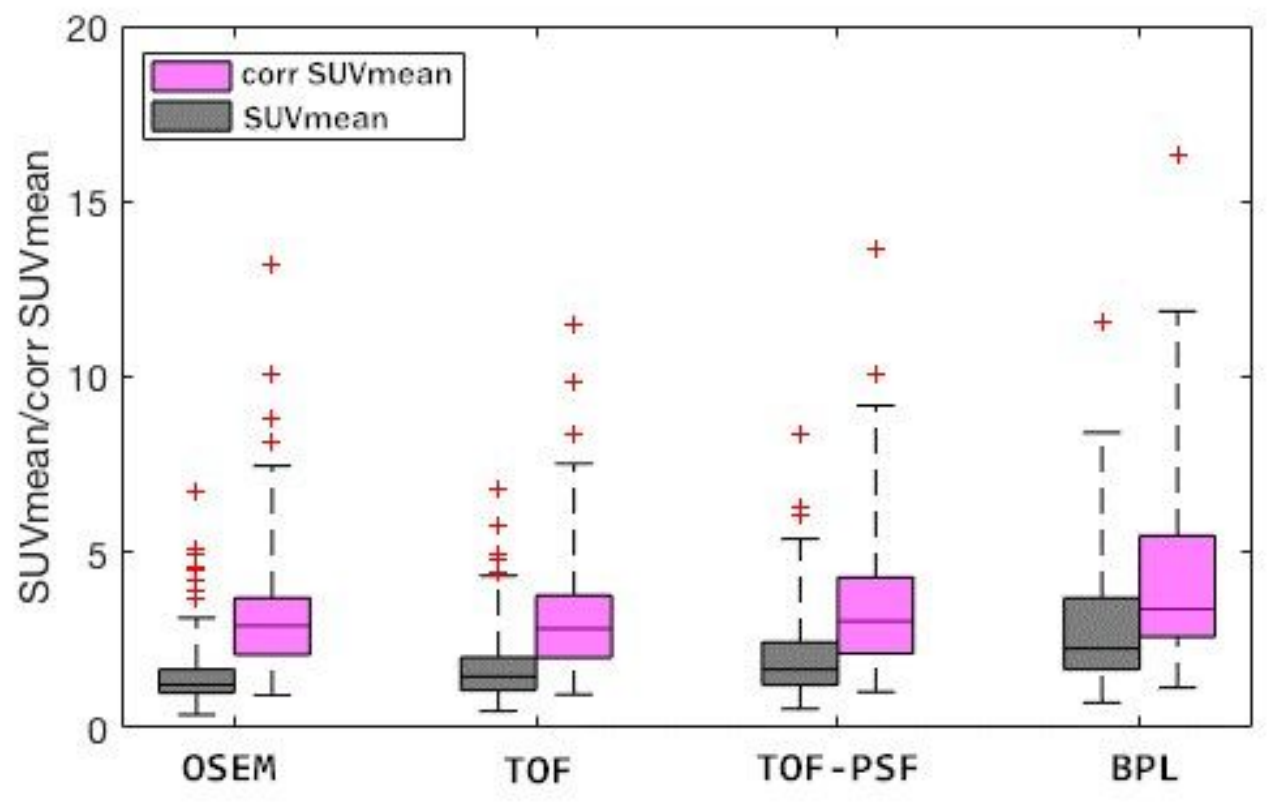

Figure 6

The box plots of SUVmean and PVE corrected SUVmean (corr SUVmean) of 75 pulmonary nodules on PET images reconstructed with different methods. After the PVE correction, the SUVmean increases represented by $\% \triangle S$ SUVmean were $124.5 \%, 85.3 \%, 77.1 \%$, and $49.4 \%$ for OSEM, TOF, TOF-PSF and BPL, respectively. 